\title{
A process-based approach to estimate point snow instability
}

\author{
B. Reuter, J. Schweizer, and A. van Herwijnen \\ WSL Institute for Snow and Avalanche Research SLF, Flüelastrasse 11, 7260 Davos Dorf, Switzerland \\ Correspondence to: B. Reuter (reuter@slf.ch)
}

Received: 1 September 2014 - Published in The Cryosphere Discuss.: 25 November 2014

Revised: 20 March 2015 - Accepted: 28 March 2015 - Published: 4 May 2015

\begin{abstract}
Snow instability data provide information about the mechanical state of the snow cover and are essential for forecasting snow avalanches. So far, direct observations of instability (recent avalanches, shooting cracks or whumpf sounds) are complemented with field tests such as the rutschblock test, since no measurement method for instability exists. We propose a new approach based on snow mechanical properties derived from the snow micropenetrometer that takes into account the two essential processes during dry-snow avalanche release: failure initiation and crack propagation. To estimate the propensity of failure initiation we define a stress-based failure criterion, whereas the propensity of crack propagation is described by the critical cut length as obtained with a propagation saw test. The input parameters include layer thickness, snow density, effective elastic modulus, strength and specific fracture energy of the weak layer - all derived from the penetration-force signal acquired with the snow micro-penetrometer. Both instability measures were validated with independent field data and correlated well with results from field tests. Comparisons with observed signs of instability clearly indicated that a snowpack is only prone to avalanche if the two separate conditions for failure initiation and crack propagation are fulfilled. To our knowledge, this is the first time that an objective method for estimating snow instability has been proposed. The approach can either be used directly based on field measurements with the snow micro-penetrometer, or be implemented in numerical snow cover models. With an objective measure of instability at hand, the problem of spatial variations of instability and its causes can now be tackled.
\end{abstract}

\section{Introduction}

Snow slope stability describes the mechanical state of the snow cover on an inclined slope and is inversely related to the probability of avalanche release (McClung and Schaerer, 2006). For a given time, depth within the snowpack, and location on a slope, snow stability can be described as the balance between snow strength and stress termed stability index (Roch, 1966). This index has been widely used (e.g., Conway and Abrahamson, 1984; Perla et al., 1982) and refined by taking into account triggering by an additional load such as a skier (Föhn, 1987). Whereas, the skier stability index has been shown to be related to the probability of skier triggering (Jamieson, 1995), this critical stress approach does not take into account that slope failure requires crack propagation. While failure initiation may depend on stress only, the propagation of cracks requires deformation energy (Bazant and Planas, 1998). Furthermore, on a slope, strength and stress are spatially variable; these variations are fundamental to the fracture process (Schweizer et al., 2003). Around locally failed areas, stress concentrations will form and drive crack propagation, and eventually cause catastrophic failure before the average material strength is reached. This observation has been termed knock-down effect (Fyffe and Zaiser, 2004) and partly explains why the stability index derived from measurements at or near natural slab avalanches often indicated stable conditions (Perla, 1977).

Not surprisingly, the link between point observations of snow stability and snow slope stability is not clear, yet (e.g., Bellaire and Schweizer, 2011). Scale issues due to different measurement scales, the so-called support and knowledge gaps between the processes involved at both scales have complicated bringing together point and slope scale snow instability results (Schweizer et al., 2008a). The point stability scale is not even well defined. Failure initiation refers to the 
collective failing of snow grains, or bonds between grains, on the scale of centimeters and the onset of a self-propagating crack in a weak snow layer called crack propagation. A common scale for both processes is the snowpack scale which spans about one square meter (Schweizer and Kronholm, 2007), which in the following we will refer to when we use the term point snow instability.

The stability index assumes a transition from stable to unstable when driving forces are no longer balanced by resisting forces. However, this approach is questionable, primarily since dry-snow slab avalanche release is the result of a series of fractures and snow properties are spatially variable. In a fracture mechanical view, to describe a material's resistance to crack propagation, flaw size and toughness need to be considered additionally to the stresses (Anderson, 1995). With the introduction of the propagation saw test (PST) (Gauthier and Jamieson, 2006; Sigrist and Schweizer, 2007) all these properties can be obtained from field data. PST experiments to study propagating cracks have confirmed deformation of the slab to substantially contribute to the mechanical energy consumed by crack extension (van Herwijnen et al., 2010). Further, Gauthier and Jamieson (2008b) have shown that the critical crack length together with the fracture result are related to slope instability. In particular, cracks propagating to the end of the column after saw cut lengths less than $50 \%$ of the column length were clear indicators of high crack propagation propensity.

There is presently no objective measurement of snow instability. Instead, recent avalanches, whumpfs or shooting cracks are considered indicators of instability (Jamieson et al., 2009), but these observations are rare. In their absence, the remaining option to gather field data on snow instability is snow instability testing (Schweizer and Jamieson, 2010). The rutschblock (RB) is a traditional snow stability test (Schweizer, 2002). The RB score was found indicative of the failure initiation propensity, the RB release type of the crack propagation propensity (Schweizer et al., 2008b). Whereas the RB release type only represents an ordinal rank, the propagation saw test (PST) gives a metric value, the critical cut length, which eases quantitative analysis. A combination of the results of both tests therefore seems appropriate for snow instability assessment.

Several studies focused on snow instability in the past, thereby either concentrating on failure initiation or crack propagation. Both, Bellaire et al. (2009) and Pielmeier and Marshall (2009) derived stability related parameters from measured snow micro-penetrometer resistance profiles. They found that weak layer strength and average slab density predicted with good accuracy stability classes estimated from RB tests.

Under the assumption of a uniform slab on a rigid substratum Heierli (2008) presented estimates of critical crack lengths obtained from recalculation of PST field experiments. Yet, averaging slab properties is a strong simplification and Schweizer (1993) pointed out the importance of slab properties for failure initiation. By means of linear elastic finite element (FE) simulations of typical snow profile types Habermann et al. (2008) found the stress at the depth of the weak layer to vary by a factor of 2 compared to a uniform slab. McClung (2009) suggested an alternative model to estimate the critical crack length by considering a finite fracture process zone.

Several numerical approaches focusing on avalanche release (for a summary see Podolskiy et al., 2013) have been made but only a few incorporate both fracture processes. Among the latest were Gaume et al. (2013) who presented a Mohr-Coulomb failure criterion based model taking into account variations of weak layer shear strength and stress redistribution by slab elasticity. Only lately, a possible refinement of the classical stability index by accounting for strength variations and their knock-down effect including a derivation of a critical crack length was presented (Gaume et al., 2014).

Predicting snow instability requires snow properties obtained either from field measurements or from snow cover modeling. In the field, the method of choice is the snow micro-penetrometer (SMP) (Schneebeli and Johnson, 1998) that allows deriving micro-structural and micro-mechanical properties from the penetration force-distance signal (Johnson and Schneebeli, 1999). Marshall and Johnson (2009) showed that values of snow density, elastic modulus and strength derived from snow micro-penetrometer signals compared well with literature data. Interpreting the oscillation of the penetration force as a Poisson shot-noise process Löwe and van Herwijnen (2012) suggested a more robust method to extract the micro-structural parameters. Their method was employed by Proksch et al. (2015) who developed a reliable parameterization of snow density applicable to a wide range of snow types. Reuter et al. (2013) showed that with the snow micro-penetrometer apart from snow density and effective modulus also the specific fracture energy of the weak layer can be derived. Comparing the results for mechanical properties obtained with snow micro-tomography (Schneebeli, 2004) to those with particle tracking velocimetry of propagation saw tests (van Herwijnen et al., 2010) they substantiated the reliability of SMP-derived parameters.

Alternatively, snow cover models provide snow structural information allowing snow instability modeling (Durand et al., 1999; Lehning et al., 2004). However, snow mechanical properties are often not simulated independently, but parameterized on density only. Schweizer et al. (2006) refined the skier's stability index implemented in the snow cover model SNOWPACK and validated it with field observations. By first identifying the potential weakness in a simulated profile and then assessing its stability. Monti et al. (2014) improved this approach to classify profiles into three classes of snow instability: poor, fair and good.

Given the fracture mechanical context of dry-snow slab avalanche release and the lack of an objective measure of instability, we propose that a description of instability 
should take into account the two essential processes in slab avalanche release, i.e., failure initiation and crack propagation, and be based on snow mechanical properties measured with the snow micro-penetrometer. Our goal is to provide an observer-independent methodology applicable to field measurements of snow stratigraphy. To this end we introduce a two-step calculation of a stability criterion and a critical crack length based on snow mechanical properties measured with the SMP. Then, we will validate the performance of our approach with field experiments of snow instability. Finally, we will show how classical snow instability observations may be interpreted in terms of failure initiation and crack propagation.

\section{Methods}

First, we present the experimental data, and then we describe how the mechanical field data acquired with the snow micropenetrometer was analyzed, before we introduce the new approach to derive snow instability.

\subsection{Field data}

Two data sets of SMP measurements were exploited to test the performance of the failure initiation (A) and the crack propagation (B) part of our approach. Data set A was originally presented by Bellaire et al. (2009). As metadata on snow instability was only available for a share of the data, 64 SMP measurements were kept for further analysis. They were all performed in close proximity $(<0.5 \mathrm{~m})$ to a RB test. The main results of a RB test, which is a point observation, are score and release type (Fig. 1). We used the score for validating the failure initiation propensity (Schweizer and Jamieson, 2010).

Data set B consists of 31 SMP measurements which have been performed in a distance less than $30 \mathrm{~cm}$ from the lower end of the column of propagation saw tests (PST) (Fig. 2). Data were collected on 7 different days. We filmed the fractures in the PSTs to precisely determine the onset of propagation by measuring the critical cut length in the pictures as a criterion of crack propagation. Both data sets also include manually observed snow profiles including snow grain type and size and hand hardness index for each manually identified layer. In addition, 77 out of the 95 field records in total contain information on either type or absence of signs of instability.

\subsection{Snow micro-penetrometer}

With the snow micro-penetrometer (SMP) a penetration resistance profile is recorded to a depth well below the weak layer at submillimeter resolution. Based on the detailed manually observed snow profile layers were defined from the corresponding sections of the signal, namely slab layers, a weak layer and a basal layer. As every layer is later represented in

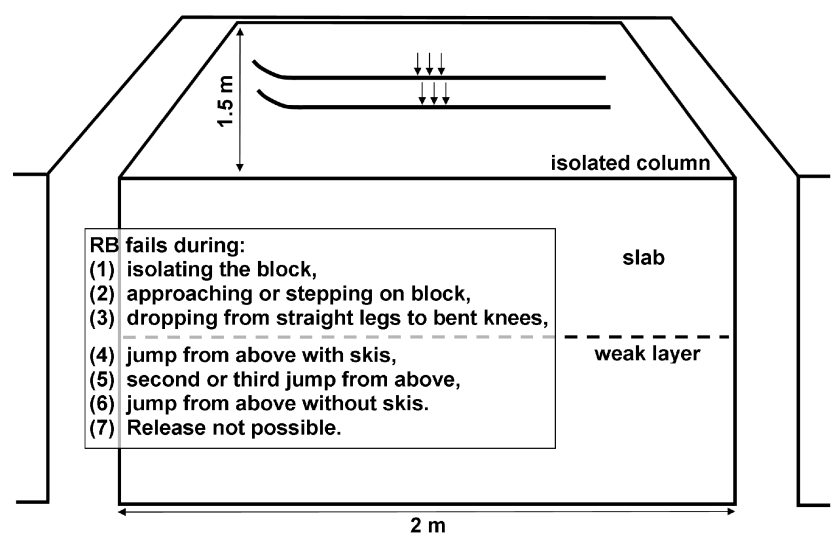

Figure 1. Sketch presenting the rutschblock (RB) test as it is seen looking upslope: after isolating a block of snow $2 \mathrm{~m}$ wide and $1.5 \mathrm{~m}$ upslope it is loaded progressively by a skier. The loading steps and scores are described in the inset. The release type was not considered here.

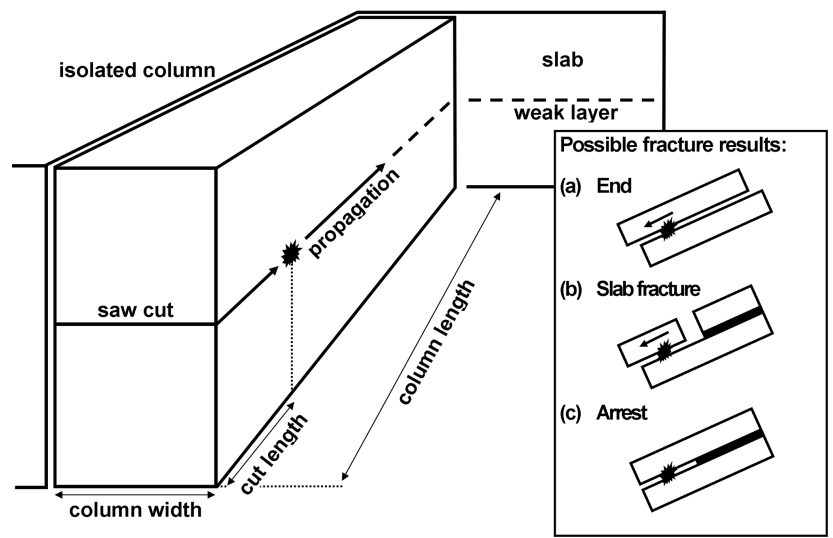

Figure 2. Sketch presenting the propagation saw test (PST) as it is seen looking upslope: after isolating a column $30 \mathrm{~cm}$ wide and at least $1.2 \mathrm{~m}$ upslope, the weak layer is cut with a snow saw from its lower end continuing upslope. Possible fracture results are described in the inset. Here, we only consider tests where the fracture went to the end of the column "End".

a finite element (FE) model and the resolution of the SMP is higher than the one needed for FE simulations, we deal with layers for the sake of shorter computation times. Figure 3 shows an example of a SMP signal with manually assigned snow layer boundaries.

Applying the shot-noise model by Löwe and van Herwijnen (2012) snow micro-structural parameters, namely the rupture force $f$, the deflection at rupture $\delta$ and the structural element size $L$ were calculated over a moving window $w$ of $2.5 \mathrm{~mm}$ with $50 \%$ overlap and then averaged over the layer. Snow density was calculated as described in Proksch et al. (2015):

$\rho=a_{1}+a_{2} \log (\widetilde{F})+a_{3} L \log (\widetilde{F})+a_{4} L$, 


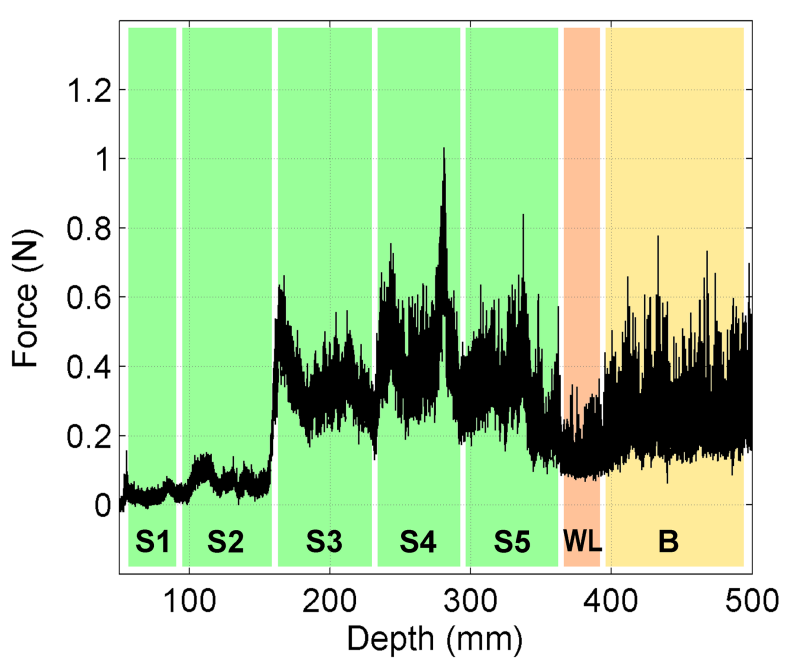

Figure 3. Penetration resistance (black) as measured with the SMP vs. snow depth. Slab layers (S1-S5) shaded in light green, weak layer (WL) shaded in light red, basal layer (B) shaded in light orange. $50 \mathrm{~mm}$ of air signal cut off.

where $a_{i}$ are coefficients, $F$ is the penetration resistance and tilde denotes the median. The micro-mechanical effective modulus and strength were calculated according to Johnson and Schneebeli (1999):

$E=\frac{f}{\delta L}$

and

$\sigma=\frac{f}{L^{2}}$.

The specific fracture energy of the weak layer (WL) was calculated as the minimum of the penetration resistance integrated across the window size $w$ within the weak layer (Reuter et al., 2013):

$w_{\mathrm{f}}=\min _{\mathrm{WL}} \int_{-\frac{w}{2}}^{+\frac{w}{2}} F \mathrm{~d} z$.

The penetration depth PS was calculated from Eq. (5) by integrating the penetration resistance $F$ from the snow surface to PS until a threshold absorbed energy $e_{\mathrm{a}}=0.036 \mathrm{~J}$ is reached. The value of $e_{\mathrm{a}}$ has been determined by comparison of SMP profiles with concurrently observed penetration depth (Schweizer and Reuter, 2015):

$e_{\mathrm{a}}=\int_{0}^{\mathrm{PS}} F(z) \mathrm{d} z$.

\subsection{Modeling}

In the following, the modeling approach to calculate estimates of the failure initiation and the crack propagation propensity of a certain slab-weak layer combination is described and validated. The mechanical properties required as input are obtained from the SMP signal as described above.

\subsubsection{Failure initiation}

A strength-over-stress criterion $S$ describes the propensity of the weak layer to fail in the case of an additional load:

$S=\frac{\sigma_{\mathrm{WL}}}{\Delta \tau}$,

with $\sigma_{\mathrm{WL}}$ being the strength of the weak layer and $\Delta \tau$ being the maximum additional shear stress at the depth of the weak layer due to skier loading. The strength of the weak layer is approximated by the micro-mechanical strength derived from the snow micro-penetrometer signal in the weak layer; i.e., we cannot use the slope-parallel shear strength because the SMP is an indentation test measuring an effective strength resulting from the mixed-mode breaking of bonds at the tip. The maximum shear stress at the depth of the weak layer was modeled with the 2-D linear elastic finite element (FE) model originally designed by Habermann et al. (2008) to calculate the shear stress at the depth of the weak layer below a layered slab due to the weight of a skier. $S$ may be interpreted as an indicator of failure initiation with low (high) values being associated with high (low) likelihood of initiating a failure. Note, the stability criterion $S$ is not expected to yield typical values of the skier's stability index ( $<1$ for "unstable", > 1.5 for "stable") (Jamieson and Johnston, 1998). One reason is that SMP-derived strength values are about 2 orders of magnitude larger than values of shear strength reported in literature (Marshall and Johnson, 2009). As the SMP measurement is a small-scale indentation test, the difference between strength values measured with the SMP and the shear frame test (Jamieson and Johnson, 2001) may be attributed to sample size and type of loading.

The 2-D FE model by Habermann et al. (2008) has been adopted to include all relevant slab layers - usually about 510 layers. The geometry of the model (Fig. 4a) was chosen such that the length of the modeled section of the snowpack $(10 \mathrm{~m})$ is at least 1 order of magnitude larger than the average depth of the weak layer to keep boundary effects small. The model consists of multiple layers including slab and basal layers as well as an embedded weak layer corresponding to the layering identified in the SMP signal. The layers are inclined by the slope angle $\alpha$. Nodes at the lower end (on the right of Fig. 4a) and at the snow-soil interface were fixed in both coordinate directions.

The model domain was divided into 2-D, quadrilateral plane strain elements having eight nodes each. The mesh consisted of 75 nodes in the horizontal and 100 nodes in the vertical per meter. The model has been implemented in ANSYS workbench to calculate the maximum shear stress within the weak layer. We assumed plane strain as stresses in the direction normal to the $x-y$ plane are smaller than within 


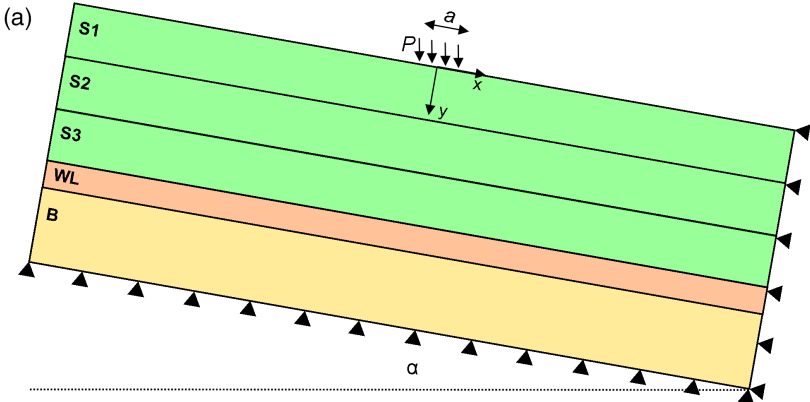

(b)

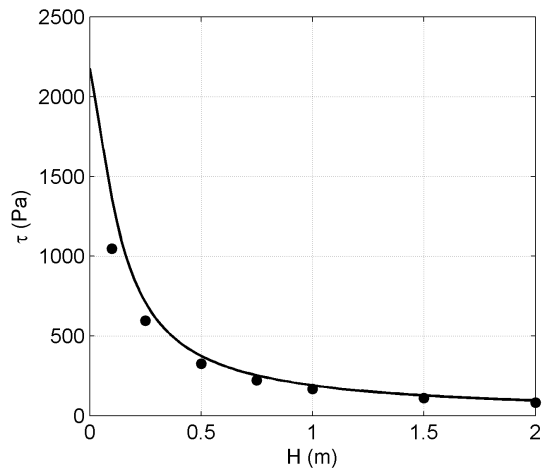

Figure 4. (a) FE model to simulate the maximum shear stress at the depth of the weak layer consisting of three slab layers (green), the weak layer (red) and a basal layer below (orange) inclined by the slope angle $\alpha$. Triangles indicate fixed nodes. The applied strip load $P$ is illustrated by black arrows pointing towards the snow surface. The axes of the coordinate system are indicated by arrows. (b) Maximum shear stress from FE simulations (dots) and from the analytical solution (line) for a uniform slab with density $200 \mathrm{~kg} \mathrm{~m}^{-3}$ and a slope angle of $38^{\circ}$ vs. slab thickness $H$.

and linear elastic behavior as the loading rate is high considering skier loading. The skier load was modeled as a static strip load $P$ of $780 \mathrm{~N}$ spread over a width $a$ of $0.2 \mathrm{~m}$. To account for skier penetration, we assumed the layers within the penetration depth to be compacted to a density of $300 \mathrm{~kg} \mathrm{~m}^{-3}$ with a corresponding elastic modulus of $16 \mathrm{MPa}$ according to Scapozza (2004); the thickness of those slab layers was adjusted so that the mass remained the same. All snow layers in the FE model were assigned thickness, density and effective modulus values as derived from the SMP signal. A fixed value of the Poisson's ratio was chosen $(v=0.25)$, as its influence is small compared to our measurement uncertainties for density or elastic modulus. From the modeled linear elastic behavior, the maximum shear stress within the weak layer was computed yielding $\Delta \tau$ of Eq. (6), i.e., not considering the stress due to the weight of the slab.

The FE model was tested to reproduce the analytical solution of McClung and Schweizer (1999) for the shear stress for a strip load on a finite area $\tau(\theta, H)$ where $\theta$ and $H$ are 2-D polar coordinates. To do so, the maximum shear stress

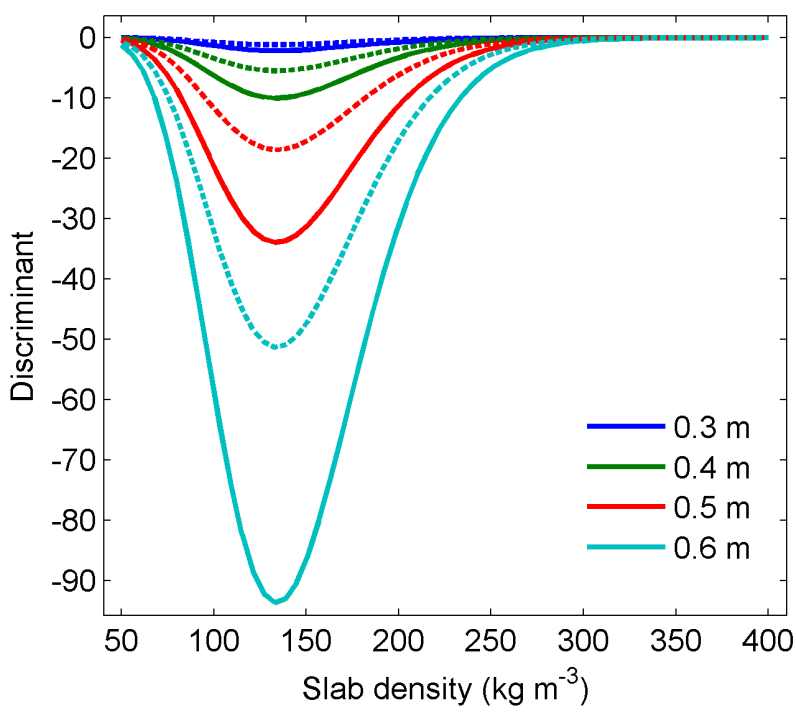

Figure 5. The polynomial's (Eq. 5) discriminant vs. slab density for typical values of slab thickness (colors); different line styles indicate flat terrain (dashed) and a slope inclined by $\alpha=38^{\circ}$ (solid lines).

at a certain depth $H$ was determined by varying $\theta$. The FE model was run with a Poisson's ratio of $v=0.49$, as the analytical solution assumes an incompressible half space. The slab was not stratified, but uniform having a density of $200 \mathrm{~kg} \mathrm{~m}^{-3}$. Hence, the solution is independent of the elastic modulus. The simulation results for different slab thickness $H$ are presented in Fig. $4 \mathrm{~b}$ together with the analytical solution. The FE model reproduced the maximum shear stress as obtained with the analytical solution very well $\left(R^{2}=0.94\right.$, regression slope $m=1.2$ ) especially for slab depth larger than the width of skier load $(0.2 \mathrm{~m})$.

\subsubsection{Crack propagation}

In order to estimate the crack propagation propensity the critical crack length as measured in a PST experiment was calculated for a weak layer embedded by a layered slab and a basal layer.

A theoretical expression (Eq. 7) linking the fracture energy of the weak layer, the elastic modulus of the slab and the critical crack length for a self-propagating crack is obtained by replacing the mechanical energy in Griffith's criterion with the total energy of the slab weak layer system found by Heierli (2008) and was presented in detail by Schweizer et al. (2011). The formulation of the total mechanical energy of the slab-weak layer system has been proven to describe the released mechanical energy of the slab in a PST reasonably well (van Herwijnen et al., 2010).

$w_{\mathrm{f}}\left(E, r_{\mathrm{c}}\right)=\frac{H}{2 E}\left[w_{0}+w_{1} \frac{r_{\mathrm{c}}}{H}+w_{2}\left(\frac{r_{\mathrm{c}}}{H}\right)^{2}+w_{3}\left(\frac{r_{\mathrm{c}}}{H}\right)^{3}+w_{4}\left(\frac{r_{\mathrm{c}}}{H}\right)^{4}\right]$, 


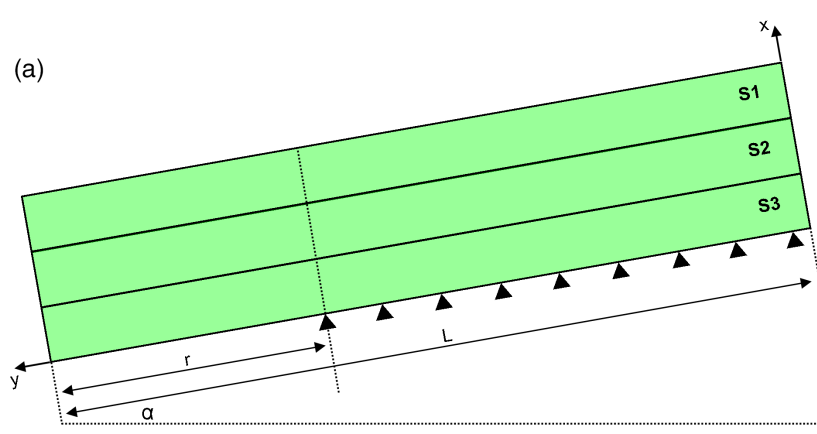

(b)

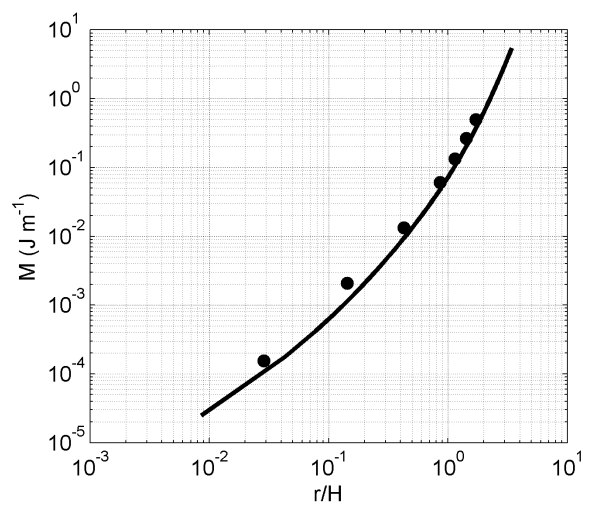

Figure 6. (a) The FE model to calculate the equivalent effective modulus contains as many slab layers as necessary to reflect the stratigraphy found in the SMP signal. Triangles indicate fixed nodes. The beam of length $L$ is overhanging a crack of length $r$ and is inclined by the slope angle $\alpha$. (b) Mechanical energy $M$ over the ratio of crack length and slab thickness $(r / H)$ modeled with FE (dots) and calculated from the analytical solution (line) for a homogeneous slab with density $200 \mathrm{~kg} \mathrm{~m}^{-3}$ and a slope angle of $30^{\circ}$.

with

$w_{0}=\frac{3 \eta^{2}}{4} \tau^{2}$

$w_{1}=\left(\pi \gamma+\frac{3 \eta}{2}\right) \tau^{2}+3 \eta^{2} \tau \sigma+\pi \gamma \sigma^{2}$,

$w_{2}=\tau^{2}+\frac{9 \eta}{2} \tau \sigma+3 \eta^{2} \sigma^{2}$

$w_{3}=3 \eta \sigma^{2}$,

$w_{4}=3 \sigma^{2}$,

and $\tau=-\rho g H \sin (\alpha)$ the shear stress, $\sigma=-\rho g H \cos (\alpha)$ the normal stress, $\gamma=1$ the elastic mismatch parameter, which is about 1 according to Heierli (2008), $\eta=$ $\sqrt{4(1+v) / 5}$ and $v=0.25$. Provided the elastic modulus $E$, the density $\rho$ and the thickness of the slab $H$, the fracture energy of the weak layer $w_{\mathrm{f}}$, and the slope angle $\alpha$ are known, the calculation of the critical crack length $r_{\mathrm{c}}$ reduces to finding the roots of Eq. (7). This fourth degree polynomial of $r_{\mathrm{c}}$ has real, ever positive coefficients. Figure 5 illustrates the dependence of the polynomial's discriminant on slab thickness and density, which is the case if a dependence of the elastic modulus on density is assumed. As the polynomial's discriminant does not change sign for typical values of density (and the elastic modulus), solutions consist of a pair of complex conjugated and two real roots. A physically meaningful solution of $r_{\mathrm{c}}$ is obtained, if the complex roots and the one with an unexpected sign are discarded.

To relax the assumption of a uniform, i.e., not stratified, slab a FE model was designed to determine the equivalent bulk modulus $E$ ' of a stratified slab (Fig. 6a). The model performed a stepwise calculation of the mechanical strain energy $M$ of a stratified slab due to bending over an increasing crack of length $r$. In order to recover an equivalent bulk modulus $E$ ', in a next step the pairs of mechanical energy and crack length $(M, r)$ were fitted with a theoretical expression of the total mechanical energy of the slab $M$ (Heierli, 2008):

$$
\begin{aligned}
M\left(E^{\prime}, r\right) & =-\frac{\pi \gamma r^{2}}{4 E^{\prime}}\left(\tau^{2}+\sigma^{2}\right) \\
& -\frac{r^{3}}{6 E^{\prime} H}\left[\lambda_{\tau \tau} \tau^{2}+\lambda_{\sigma \tau} \tau \sigma+\lambda_{\sigma \sigma} \sigma^{2}\right],
\end{aligned}
$$

with

$$
\begin{aligned}
& \lambda_{\tau \tau}=1+\frac{9}{4} \eta\left(\frac{r}{H}\right)^{-1}+\frac{9}{4} \eta^{2}\left(\frac{r}{H}\right)^{-2} \\
& \lambda_{\tau \sigma}=\frac{9}{2} \eta+\frac{9}{2} \eta^{2}\left(\frac{r}{H}\right)^{-1} \\
& \lambda_{\sigma \sigma}=3 \eta^{2}+\frac{9}{4} \eta \frac{r}{H}+\frac{9}{5}\left(\frac{r}{H}\right)^{2} .
\end{aligned}
$$

The FE model consists of stratified layers, which were assigned SMP-derived values of density, effective modulus and thickness (Fig. 6a). The Poisson's ratio was kept constant ( $v=0.25$ ). Due to its geometry (only considering slab layers) and boundary conditions (rigid support along the ligament length $(L-r))$, the FE model only considers the behavior of the slab layers as described with the formulation of the total mechanical energy of the slab-weak layer system, neglecting deformation in the weak or basal layers. In our model, the deflecting beam never got in touch with the basal layer, which, however, may be the case in field experiments, in particular with soft slabs. The FE model reproduced the theoretical formulation very well $\left(R^{2}=0.85\right)$, especially for crack lengths $r$ greater or equal the thickness of the overlying slab $H$ (Fig. 6b). With the bulk equivalent modulus $E$ ', we find the exact solution of Eq. (7) and obtain the critical crack length $r_{\mathrm{c}}$ for the specific slab-weak layer combination.

\section{Results}

In the following, both model parts predicting the propensity of the snowpack to failure initiation and crack propagation are evaluated with the two independent data sets (A and B). 


\subsection{Failure initiation}

For each of the 66 SMP profiles with corresponding RB test (data set A) the failure initiation criterion $S$ was calculated. SMP-derived density, effective modulus, strength and layer thickness were used to drive the FE model. For the comparison with the RB score, we grouped scores 1 and 2 as well as 6 and 7 because scores 1 and 7 were observed infrequently. The criterion $S$ increased with increasing RB score (Fig. 7a). If for a given $S$ there was no overlap of the boxes, the predictive power of $S$ would obviously be very good. Although this is not the case, the medians of the failure initiation criterion (indicated by gray lines) per RB score increased monotonically with increasing RB scores. This monotonic increase is reflected in a high Spearman rank correlation coefficient $\left(r_{\mathrm{s}}>0.9\right)$. If results are grouped by scores in two stability classes of $\mathrm{RB}<4$ and $\mathrm{RB} \geq 4$, a threshold previously found to separate lower and higher stability (e.g., Schweizer and Jamieson, 2003), the criterion $S$ discriminated well between the two classes (Wilcoxon rank sum test, level of significance $p=0.01$ ) with a classification tree splitting value of $S=133$.

\subsection{Crack propagation}

All 31 SMP signals from data set B were analyzed and the critical cut length $r_{\mathrm{c}}$ was calculated from Eq. (7) with SMPderived mechanical properties being density, effective modulus, specific fracture energy and layer thickness. In Fig. 8 the results are contrasted with the critical crack lengths measured in the field in the PST experiments adjacent to the SMP measurements. On the left, (Fig. 8a) model results are shown for the case of a uniform slab; i.e., density and effective modulus were averaged to show the effect of neglecting the stratigraphy of the slab. Modeled values overestimated the critical cut length yielding a rather fair Pearson correlation coefficient of $r_{\mathrm{p}}=0.58$ and a coefficient of determination of $R^{2}=0.29$. Only for a few experiments were modeled and observed crack lengths similar, indicating that assuming a uniform slab is not a good approximation. In fact, Fig. $8 \mathrm{~b}$ shows that the agreement between model results and observations improved if the stratification of the slab was taken into account. All identified slab layers were assigned the corresponding density and effective modulus obtained from SMP signal processing and input in the FE model to determine the bulk effective modulus of the slab. The modeled values of critical crack length were clearly related to the measured values $\left(r_{\mathrm{p}}=0.83\right)$ as indicated by the collapse of the linear regression on the $1: 1$ line (Fig. 8b). The regression slope was well-defined $(p<0.01)$ with some scatter $\left(R^{2}=0.50\right)$ indicating the uncertainty involved with the presented approach. The critical crack length was predicted with a root mean squared error of $2 \mathrm{~cm}$, a mean absolute error of $7 \mathrm{~cm}$ and a mean absolute percentage error of $9 \%$.
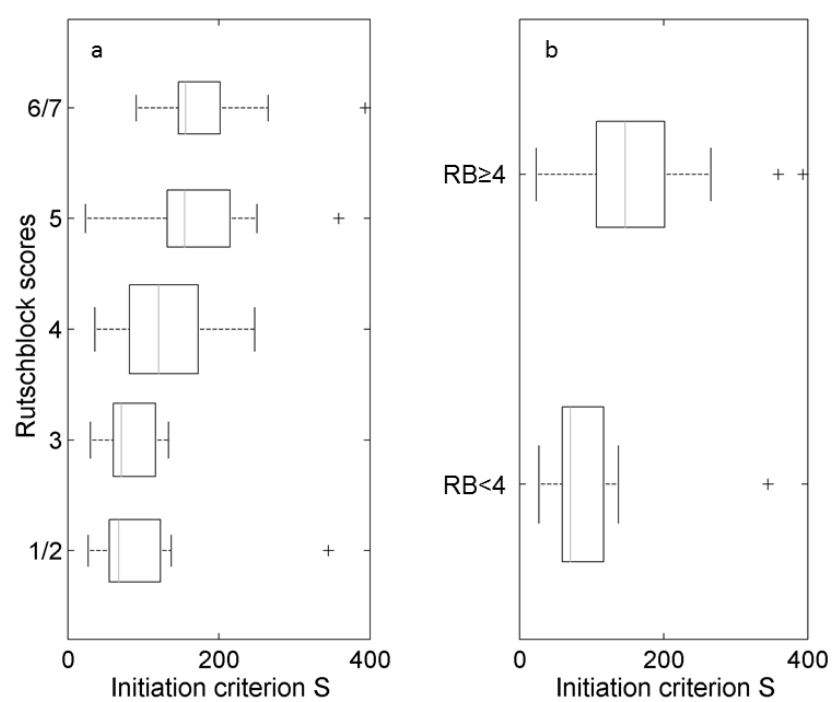

Figure 7. Modeled failure initiation criterion $S$ (a) vs. RB score and (b) vs. RB stability classes: $\mathrm{RB}<4(N=38)$ and $\mathrm{RB} \geq 4(N=26)$. Boxes span the interquartile range from 1 st to 3 rd quartile with a horizontal line showing the median (grey line). Widths of the boxes correspond to the number of cases. Whiskers extend to the most extreme data points not considered outliers (crosses) within 1.5 times the interquartile range above the $3 \mathrm{rd}$ and below the 1 st quartile.

\subsection{Validation with signs of instability}

Model results were further compared with independent field observations of signs of instability such as whumpfs, shooting cracks and recent avalanches. Both data sets (A and B) included records of such field observations which we grouped in three categories: whumpfs, shooting cracks with or without whumpfs ("cracks") or "all signs" (whumpfs, cracks and recent avalanches); i.e., fresh avalanches were only observed simultaneously with whumpfs and cracks (Fig. 9). To jointly relate our modeled estimates of instability to the observations of instability we contrasted the propensity to crack propagation, i.e., modeled critical crack length, and failure initiation, i.e., initiation criterion $S$, in Fig. 9. Signs of instability were primarily present in the lower left of Fig. 9, i.e., for low values of the failure initiation criterion and the critical crack length. Vice versa no signs of instability were reported if both criteria yielded high values (upper right). This finding suggests that both criteria, the one for failure initiation and the one for crack propagation, are linked to snow instability. A classification tree with the two independent variables $S$ and $r_{\mathrm{c}}$ yielded splits of $S=234$ and $r_{\mathrm{c}}=0.41 \mathrm{~m}$ which separate between the cases with and without concurrently observed signs of instability (Fig. 9). These thresholds divide the plot into four quadrants. In the lower left quadrant all 35 cases with signs of instability as well as 10 cases without signs of instability were found. Our split value $(S=234)$ for the initiation criterion $S$ is very similar to the one found by Schweizer and Reuter (2015) who reported a value of 212. 

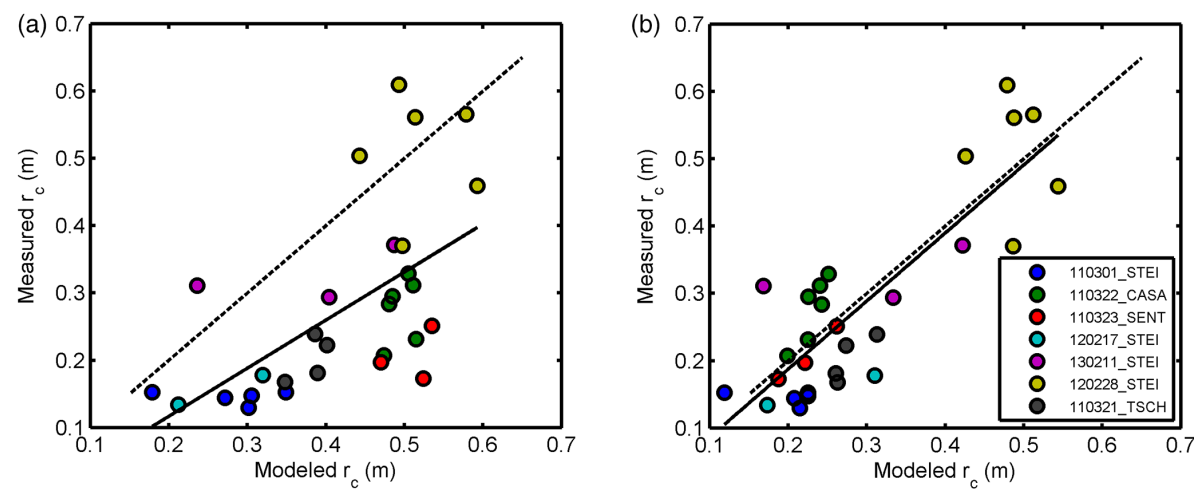

Figure 8. Critical crack lengths $r_{\mathrm{c}}$ predicted from Eq. (7) are contrasted with critical crack lengths measured in the field ( $\left.N=31\right)$. Experiments grouped by date and location with colors. Solid line shows linear regression, dashed line indicates the 1: 1 line. (a) Slab stratigraphy neglected (average density, average effective modulus). (b) Density and effective modulus of each snow layer taken into account by FE simulation.

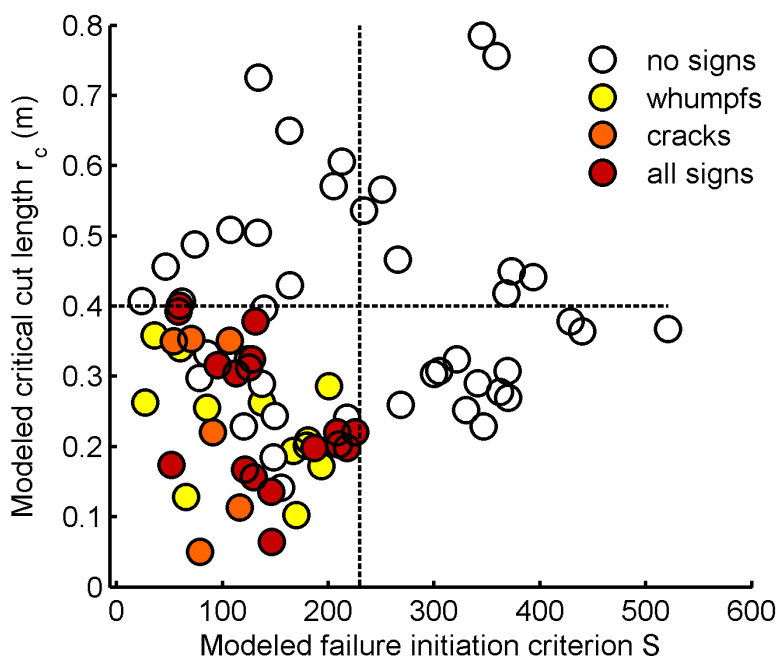

Figure 9. Type and presence of signs of instability against failure initiation criterion $S$ and critical crack length $r_{\mathrm{c}}$, both modeled, for data sets $\mathrm{A}$ and $\mathrm{B}$, if reported $(N=77)$. Colors indicate type of observed signs of instability: whumpfs, shooting cracks with or without whumpfs (cracks) or all signs (whumpfs, cracks and recent avalanches observed). Open circles indicate that no signs of instability were reported explicitly (no signs). Dashed lines represent split values dividing the plot into four quadrants as found with a classification tree.

In regard to the modeled critical crack length, Gauthier and Jamieson (2008a) suggested a value of $<50 \%$ of the column length which in their study corresponded to $50 \mathrm{~cm}$. Assuming crack propagation to be likely (two lower quadrants) or failure initiation to be easy (two left quadrants) does not distinguish sharply between signs of instability present or absent. However, if both criteria had low values unstable snow conditions were observed (lower left quadrant).

\section{Discussion}

In our present understanding avalanche release is seen as a sequence of fractures. To capture the two most important steps preceding the detachment of a snow slab we addressed the stress at the depth of a potential weakness with the failure initiation criterion $S$ and the critical crack size for selfpropagation with the critical crack length $r_{\mathrm{c}}$. We presented a model approach to derive both quantities from snow micropenetrometer signals which is a fast method to acquire information on mechanical properties in the field.

Assessing the performance of the model approach with two different field tests (RB and PST) yielded plausible results. However, the main source of uncertainty is related to the mechanical properties needed as input for the model. Snow density, effective modulus and specific fracture energy were all determined from SMP measurements. Uncertainties related to the determination of these mechanical properties have recently been addressed by Proksch et al. (2015) and Reuter et al. (2013) and lie within 10-20\% for density and fracture energy. Other SMP error sources are known, so erroneous signals were identified and discarded. Some errors were user-related such as mechanical disturbances. Other unavoidable errors such as signal drift due to strong temperature changes in the snowpack or stick slip of the rod at high snow densities were rare.

The SMP-derived failure initiation criterion $S$ performed well based on the evaluation with rutschblock tests, yielding a better correlation than the one lately observed by Schweizer and Reuter (2015) using the compression test. They concluded that the dimensions of the compression test and the type of loading are not ideal for modeling purposes. While the RB test includes six different loading steps, the load is only increased twice in a compression test, but numerous taps are performed within the same loading range. The loading of the RB and consequently the stress exerted on the weak layer increases monotonically with the score (score four and five 
have the same load). This is reflected in the fair discrimination of RB scores four and five with the failure initiation criterion $S$. Furthermore, RB loading steps are ordinal numbers; i.e., they can be ranked, but they do not follow a known relation with stability. Hence, the stress in the weak layer increases stepwise in the experiment, whereas the modeled stability is continuous. The box plots in Fig. 7 group modeled values of failure initiation $(S)$ with rutschblock classes. The monotonic increase of the medians suggests that the criterion $S$ reflects the propensity of failure initiation in a weak layer below a layered slab. Correlations of the rutschblock release type were neither significant with the initiation criterion $S\left(r_{\mathrm{s}}=0.11, p=0.39\right)$, nor with the modeled critical cut length $\left(r_{\mathrm{s}}=0.04, p=0.76\right)$.

The critical cut length was modeled with an accuracy of a few centimeters (RMSE of $2 \mathrm{~cm}$ ). It was shown that the slab layering played an important role in the process of crack propagation. Only with the introduction of the bulk effective modulus imitating the bending behavior of a layered slab measured critical cut lengths were reproduced with good accuracy (Fig. 8). Until now, research on snow instability had mainly focused on weak layer or average slab properties (Bellaire et al., 2009; Pielmeier and Marshall, 2009), and field studies quantifying the influence of snow layering on snow instability (Reuter and Schweizer, 2012) were rare. Alternatively, the critical value of the crack length could have been determined by stepwise increasing the crack length in an FE model until the critical energy release rate reaches the specific fracture energy of the weak layer. This approach, comparable to that of Mahajan and Joshi (2008), however, was not followed due to its high computational expenses, as repeated meshing for every single iteration step would be costly.

The introduced FE models assumed linear elastic behavior and were confined to two dimensions. These assumptions are in contrast with our knowledge that snow is a porous medium consisting of a non-isotropic ice/air matrix, exhibiting plastic, elastic and viscous behavior at the macro scale. However, as loading rates in RB tests and PSTs are high, linear elastic assumptions are justified - for the rutschblock test at least at a certain depth below the snow surface. Two-dimensional modeling seems sufficient, as three-dimensional modeling is not advantageous due to the lack of experimental orthotropic material properties at this point of time.

\section{Conclusions}

We have developed a novel approach to determine quantitative estimates of both, the failure initiation and crack propagation propensity of the snowpack based on mechanical properties derived from objective snow micro-penetrometer measurements. Based on the current understanding of drysnow slab avalanche release it includes the mechanical properties of all relevant layers embedding the weak layer to make predictions on the propensity of initiating a failure and spreading the crack in a weak layer within the snowpack. The presented approach is process-based, observer-independent and relies on measurements of mechanical properties.

The performance of the two novel measures of instability has been assessed in comparisons with two different data sets of field tests (rutschblock and propagation saw test). Both measures of instability, the stress criterion $S$ as well as the critical crack length $r_{\mathrm{c}}$ were well correlated with the results of field tests. In addition, the importance of slab layering especially with respect to crack propagation has been shown. The comparison of our modeled estimates of snow instability with field observations of signs of instability clearly indicated that a snowpack is unstable only in case of high failure initiation as well as high crack propagation propensity. Although we anticipated this finding, i.e., that both conditions have to be fulfilled, we are not aware, to the best of our knowledge, that it has been demonstrated before.

Recent field studies have frequently focused on identifying spatial variations of snow instability and its drivers, which requires an objective measure of instability, which was so far lacking. With the observer-independent method (we presented taking into account both failure initiation and crack propagation processes) it will become possible to resolve causes of spatial snow instability variations. With respect to operational application in the context of avalanche forecasting, our approach based on field measurements could be employed, provided a robust and reliable snow micropenetrometer is at hand which additionally allows remote data transfer and automatic processing, or be implemented in numerical snow cover models.

Acknowledgements. B. Reuter has been supported by a grant of the Swiss National Science Foundation (200021_144392). We thank numerous colleagues from SLF for help with the field work. We are grateful for the constructive review comments by E. Podolskiy and B. Jamieson.

Edited by: F. Dominé

\section{References}

Anderson, T. L.: Fracture Mechanics: Fundamentals and Applications, CRC Press, Boca Raton, USA, 1995.

Bazant, Z. P. and Planas, J.: Fracture and size effect in concrete and other quasibrittle materials, CRC Press, Boca Raton, USA, 1998.

Bellaire, S. and Schweizer, J.: Measuring spatial variations of weak layer and slab properties with regard to snow slope stability, Cold Reg. Sci. Technol., 65, 234-241, 2011.

Bellaire, S., Pielmeier, C., Schneebeli, M., and Schweizer, J.: Stability algorithm for snow micropenetrometer measurements, J. Glaciol., 55, 805-813, 2009. 
Conway, H. and Abrahamson, J.: Snow stability index, J. Glaciol., 30, 321-327, 1984.

Durand, Y., Giraud, G., Brun, E., Mérindol, L., and Martin, E.: A computer-based system simulating snowpack structures as a tool for regional avalanche forecasting, J. Glaciol., 45, 469-484, 1999.

Föhn, P. M. B.: The stability index and various triggering mechanisms, in: Symposium at Davos 1986 - Avalanche Formation, Movement and Effects, IAHS Publ. 162, edited by: Salm, B. and Gubler, H., International Association of Hydrological Sciences, Wallingford, Oxfordshire, UK, 195-214, 1987.

Fyffe, B. and Zaiser, M.: The effects of snow variability on slab avalanche release, Cold Reg. Sci. Technol., 40, 229-242, 2004.

Gaume, J., Chambon, G., Eckert, N., and Naaim, M.: Influence of weak-layer heterogeneity on snow slab avalanche release: application to the evaluation of avalanche release depths, J. Glaciol., 59, 423-437, 2013.

Gaume, J., Schweizer, J., van Herwijnen, A., Chambon, G., Reuter, B., Eckert, N., and Naaim, M.: Evaluation of slope stability with respect to snowpack spatial variability, J. Geophys. Res., 119, 1783-1799, doi:10.1002/2014JF003193, 2014.

Gauthier, D. and Jamieson, B.: Towards a field test for fracture propagation propensity in weak snowpack layers, J. Glaciol., 52, 164 168, doi:10.3189/172756506781828962, 2006.

Gauthier, D. and Jamieson, B.: Evaluation of a prototype field test for fracture and failure propagation propensity in weak snowpack layers, Cold Reg. Sci. Technol., 51, 87-97, 2008a.

Gauthier, D. and Jamieson, B.: Fracture propagation propensity in relation to snow slab avalanche release: validating the Propagation Saw Test, Geophys. Res. Lett., 35, L13501, doi:10.1029/2008GL034245, 2008b.

Habermann, M., Schweizer, J., and Jamieson, J. B.: Influence of snowpack layering on humantriggered snow slab avalanche release, Cold Reg. Sci. Technol., 54, 176-182, 2008.

Heierli, J.: Anticrack model for slab avalanche release, $\mathrm{PhD}$ thesis, University of Karlsruhe, Karlsruhe, Germany, 102 pp., 2008.

Jamieson, B., Haegeli, P., and Schweizer, J.: Field observations for estimating the local avalanche danger in the Columbia Mountains of Canada, Cold Reg. Sci. Technol., 58, 84-91, 2009.

Jamieson, J. B.: Avalanche prediction for persistent snow slabs, $\mathrm{PhD}$ thesis, Department of Civil Engineering, University of Calgary, Calgary AB, Canada, 258 pp., 1995.

Jamieson, J. B. and Johnston, C. D.: Refinements to the stability index for skier-triggered dry slab avalanches, Ann. Glaciol., 26, 296-302, 1998.

Jamieson, J. B. and Johnston, C. D.: Evaluation of the shear frame test for weak snowpack layers, Ann. Glaciol., 32, 59-68, 2001.

Johnson, J. B. and Schneebeli, M.: Characterizing the microstructural and micromechanical properties of snow, Cold Reg. Sci. Technol., 30, 91-100, 1999.

Lehning, M., Fierz, C., Brown, R. L., and Jamieson, J. B.: Modeling instability for the snow cover model SNOWPACK, Ann. Glaciol., 38, 331-338, 2004.

Löwe, H. and van Herwijnen, A.: A poisson shot noise model for micro-penetration of snow, Cold Reg. Sci. Technol., 70, 62-70, 2012.

Mahajan, P. and Joshi, S. K.: Modeling of interfacial crack velocities in snow, Cold Reg. Sci. Technol., 51, 98-111, 2008.
Marshall, H.-P. and Johnson, J. B.: Accurate inversion of highresolution snow penetrometer signals for microstructural and micromechanical properties, J. Geophys. Res., 114, F04016, doi:10.1029/2009JF001269, 2009.

McClung, D. M.: Dry snow slab quasi-brittle fracture initiation and verification from field tests, J. Geophys. Res., 114, F01022, doi:10.1029/2007JF000913, 2009.

McClung, D. M. and Schaerer, P.: The Avalanche Handbook, The Mountaineers Books, Seattle WA, USA, 2006.

McClung, D. M. and Schweizer, J.: Skier triggering, snow temperatures and the stability index for dry slab avalanche initiation, J. Glaciol., 45, 190-200, 1999.

Monti, F., Schweizer, J., and Gaume, J.: Deriving snow stability information from simulated snow cover stratigraphy, in: Proceedings ISSW 2014, International Snow Science Workshop, 29 September-3 October 2014, Banff, Alberta, Canada, 465469, 2014.

Perla, R.: Slab avalanche measurements, Can. Geotech. J., 14, 206213, 1977.

Perla, R., Beck, T. M. H., and Cheng, T. T.: The shear strength index of alpine snow, Cold Reg. Sci. Technol., 6, 11-20, 1982.

Pielmeier, C. and Marshall, H.-P.: Rutschblock-scale snowpack stability derived from multiple quality-controlled SnowMicroPen measurements, Cold Reg. Sci. Technol., 59, 178-184, 102009.

Podolskiy, E. A., Chambon, G., Naaim, M., and Gaume, J.: A review of finite-element modelling in snow mechanics, J. Glaciol., 59, 1189-1201, 2013.

Proksch, M., Löwe, H., and Schneebeli, M.: Density, specific surface area, and correlation length of snow measured by highresolution penetrometry, J. Geophys. Res.-Earth, 120, 346-362, doi:10.1002/2014JF003266, 2015.

Reuter, B. and Schweizer, J.: The effect of surface warming on slab stiffness and the fracture behavior of snow, Cold Reg. Sci. Technol., 83-84, 30-36, 2012.

Reuter, B., Proksch, M., Loewe, H., van Herwijnen, A., and Schweizer, J.: On how to measure snow mechanical properties relevant to slab avalanche release, in: Proceedings ISSW 2013, International Snow Science Workshop, 7-11 October 2013, Grenoble, France, 38-42, 007-011, 2013.

Roch, A.: Les déclenchements d'avalanches, in: Symposium at Davos 1965 - Scientific Aspects of Snow and Ice Avalanches, IAHS Publication 69, Int. Assoc. Hydrol. Sci., Wallingford, UK, 182-197, 1966 (in French).

Scapozza, C.: Entwicklung eines dichte- und temperaturabhängigen Stoffgesetzes zur Beschreibung des visko-elastischen Verhaltens von Schnee, PhD thesis, Institut für Geotechnik, ETH Zurich, Zurich, Switzerland, 250 pp., 2004 (in German).

Schneebeli, M.: Numerical simulation of elastic stress in the microstructure of snow, Ann. Glaciol., 38, 339-342, 2004.

Schneebeli, M. and Johnson, J. B.: A constant-speed penetrometer for high-resolution snow stratigraphy, Ann. Glaciol., 26, 107111, 1998.

Schweizer, J.: The influence of the layered character of snow cover on the triggering of slab avalanches, Ann. Glaciol., 18, 193-198, 1993.

Schweizer, J.: The Rutschblock test - procedure and application in Switzerland, The Avalanche Review, 20, 14-15, 2002.

Schweizer, J. and Jamieson, J. B.: Snowpack properties for snow profile analysis, Cold Reg. Sci. Technol., 37, 233-241, 2003. 
Schweizer, J. and Jamieson, J. B.: Snowpack tests for assessing snow-slope instability, Ann. Glaciol., 51, 187-194, 2010.

Schweizer, J. and Kronholm, K.: Snow cover spatial variability at multiple scales: characteristics of a layer of buried surface hoar, Cold Reg. Sci. Technol., 47, 207-223, 2007.

Schweizer, J. and Reuter, B.: A new index combining weak layer and slab properties for snow instability prediction, Nat. Hazards Earth Syst. Sci., 15, 109-118, doi:10.5194/nhess-15-109-2015, 2015.

Schweizer, J., Jamieson, J. B., and Schneebeli, M.: Snow avalanche formation, Rev. Geophys., 41, 1016, doi:10.1029/2002RG000123, 2003.

Schweizer, J., Bellaire, S., Fierz, C., Lehning, M., and Pielmeier, C.: Evaluating and improving the stability predictions of the snow cover model SNOWPACK, Cold Reg. Sci. Technol., 46, 52-59, 2006.

Schweizer, J., Kronholm, K., Jamieson, J. B., and Birkeland, K. W.: Review of spatial variability of snowpack properties and its importance for avalanche formation, Cold Reg. Sci. Technol., 51, 253-272, 2008a.
Schweizer, J., McCammon, I., and Jamieson, J. B.: Snowpack observations and fracture concepts for skier-triggering of dry-snow slab avalanches, Cold Reg. Sci. Technol., 51, 112-121, 2008b.

Schweizer, J., van Herwijnen, A., and Reuter, B.: Measurements of weak layer fracture energy, Cold Reg. Sci. Technol., 69, 139144, 2011.

Sigrist, C. and Schweizer, J.: Critical energy release rates of weak snowpack layers determined in field experiments, Geophys. Res. Lett., 34, L03502, doi:10.1029/2006GL028576, 2007.

van Herwijnen, A., Schweizer, J., and Heierli, J.: Measurement of the deformation field associated with fracture propagation in weak snowpack layers, J. Geophys. Res., 115, F03042, doi:10.1029/2009JF001515, 2010. 